\title{
Exploraciones en torno a los conceptos de Rodolfo Kusch, el pensamiento nacional y los levantamientos populares latinoamericanos*
}

\author{
Approaches in Kusch conceptualization, national thought and Latino \\ American popular uprinsings
}

Por: Pageau, Christian

Román. Maximiliano

Sánchez Blanco, Ricardo Alejandro*

Universidad Nacional del Nordeste-UNNE -

Argentina

Email: pageau.christian@gmail.com, ORCID 0000-0002-2475-7855

maxiroman@hotmail.com

ricardoalejandrosanchezblanco@gmail.com

Fecha de recepción: 19/03/2021

Fecha de aprobación: 30/05/2021

DOI: http://dx.doi.org/10.30972/nvt.1715352

\section{Resumen}

Nuestra reflexión común -y diferenciada por la formación respectiva- se interesa en pensar otras vías de entradas a la comprensión acerca de la naturaleza de nuestra América, su cultura, su historia y sus luchas políticas populares. Presentamos

\footnotetext{
* Los autores forman parte del Proyecto de Investigación “Manifestaciones y narrativas interdisciplinarias en Argentina: literatura, artes y cultura" PI $18 \mathrm{H} 002$ (Secretaría General de Ciencia y Técnica - Universidad Nacional del Nordeste)

* Christian Pageau es Doctor en Letras por la UNNE y docente en Humanidades, UNNE. Investiga literatura y pensamiento latinoamericano, especialmente desde la perspectiva descolonial. Es detentor de una maestría y título de grado en Estudios Hispánicos, por la Universidad de Montreal. Es ciudadano canadiense, y argentino naturalizado desde 2016.

Ricardo Alejandro Sánchez Blanco es Licenciado y Profesor en Filosofía por la UNNE y docente en la Facultad de Ciencias Económicas de la UNNE. Investiga el pensamiento argentino y latinoamericano en cuanto a su problemática filosófica y política.

Maximiliano Román es Profesor en Filosofía por la UNNE y docente en la Facultad de Humanidades y en la Facultad de Ciencia Exactas, Naturales y Agrimensura de la UNNE. Investiga sobre las características locales de la conflictividad social y sobre los marcos conceptuales para su estudio. Coordina el Observatorio de Conflictos Sociales del Nordeste Argentino, desde el cual se articulan prácticas de extensión y articulación con organizaciones sociales y políticas.
} 
Exploraciones en torno a los conceptos de Rodolfo Kusch, el pensamiento nacional y los levantamientos populares latinoamericanos

primero las categorías kuscheanas en su contexto filosófico, para luego estudiar brevemente el modo en que permiten pensar de modo original la cuestión del ser nacional y del colonialismo interno en el pensamiento de J. J. Hernández Arregui, tanto como la posible descolonización de la mirada a los movimientos populares americanos del presente siglo. Concluimos, provisionalmente, que las categorías sentipensantes de Kusch, por la ruptura epistémica que suponen, permiten caracterizar mejor la opción por el pueblo y los modos de entender de otra manera los contextos del neocolonialismo. Finalmente, pensar la lucha popular desde la categoría de fagocitación permite hacer surgir la fundamental característica comunal de estos movimientos políticos.

Palabras clave: Hedor; Pulcritud; Ser; Estar; Fagocitación.

\section{Abstract}

Our common reflection -and differentiated by the respective formation- is interested in thinking of other ways of entering the understanding about the nature of our America, its culture, its history and its popular political struggles. We present first the Kuschean categories in their philosophical context, and then briefly study the way in which they allow to think in an original way the question of national-being and of internal colonialism in the thought of J.J. Hernández Arregui, as well as the possible decolonization of the gaze on the American popular movements of this century. We conclude, provisionally, that the decolonial categories of Kusch, by the epistemic rupture that they suppose, allow to characterize better the option for the people and the ways of understanding in another way the contexts of neo-colonialism. Finally, thinking about the popular struggle from the category of phagocytosis allows to highlight the fundamental communal characteristic of these political movements.

Keywords: Stench; Pulchritude; Becoming; Being; Phagocytosis

\section{Cómo citar este artículo:}

APA: Pageau et al. (2021). Exploraciones en torno a los conceptos de Rodolfo Kusch, el pensamiento nacional y los levantamientos populares latinoamericanos. Nuevo Itinerario, 17 (1), 139-165. 
En Nuestra América -retomando el concepto de José Martí-, desde los comienzos mismos de la Conquista, varios discursos y luchas se han conformado desde los márgenes en una continua lucha por la construcción de un lugar de enunciación y de reflexión propio, situado, atento a la experiencia popular en su diversidad, contra las censuras propias y ajenas, en reacción al pachakuti epistémico que implicó el inicio de la modernidad colonial eurocentrada. Desde la filosofía y la literatura, tales son nuestros campos de formación y actuación docente e investigativa, nos reconocemos en el sentipensar situado del filósofo argentino Rodolfo G. Kusch. La ruptura que este autor logra instalar en los modos eurocentrados, colonizantes, de pensar nuestra América y sus experiencias culturales y populares, su forma de indagar, de modo sensible e intuitivo, en la honda y misteriosa alma de Nuestra América Profunda, nos aparece una posible fuente generadora de nuevas miradas en nuestros respectivos campos de investigación. Este artículo pretende plasmar algunas exploraciones iniciadas en ese sentido.

En un primer momento, presentaremos las categorías analíticas que propone Kusch desde 1963 en su obra América Profunda. Es decir, el hedor, la pulcritud, el ser, el estar y la fagocitación, pues son las que guían nuestras reflexiones. Entendemos que estos conceptos configuran una perspectiva original, elaborada a partir del intercambio con el pensamiento popular americano, que permite una reflexión situada sobre múltiples ámbitos de la realidad. En un segundo momento, indagaremos en algunas propuestas del intelectual argentino Juan José Hernández Arregui, especialmente acerca del ser nacional y el colonialismo interno. Su articulación con el sistema categorial de Kusch es potencialmente fecunda para develar el lado oscuro de la modernidad en nuestras tierras y las consecuencias de la herencia colonial en sus diversas manifestaciones. Finalmente, intentamos operativizar los conceptos kuscheanos para proponer elementos que permitan descolonizar la mirada sobre los levantamientos protagonizados por los pueblos latinoamericanos durante las últimas décadas, enfocando en las experiencias y conocimientos de las comunidades de lucha.

1. Rodolfo G. Kusch y la búsqueda de una Filosofía Latinoamericana 
Exploraciones en torno a los conceptos de Rodolfo Kusch, el pensamiento nacional y los levantamientos populares latinoamericanos

Rodolfo Gunther Kusch (1922-1979) fue un filósofo argentino que plasmó con su obra, y también con su trayectoria vital, la búsqueda incesante de un filosofar latinoamericano. Fue pionero en plantear, ya en sus primeros escritos filosóficos, la necesidad de introducirse profundamente en el "ser americano" desde el diálogo con las culturas originarias y centrar el pensar en nosotres mismes o, más bien, en un autoreconocimiento como sujetos latinoamericanos contextualizados y arraigados. De allí su crítica frontal a la filosofía académica y la presunción de que existe una forma otra de pensamiento popular:

En materia de filosofía tenemos en América, por una parte, una forma oficial de tratarla y, por la otra, una forma, por decir así, privada de hacerlo. Por un lado, está la que aprendemos en la universidad y que consiste en una problemática europea traducida a nivel filosófico y, por el otro, un pensar implícito vivido cotidianamente en la calle o en el campo (Kusch, 2007, p. 263).

Ahora bien, para Kusch, reflexionar sobre lo latinoamericano implica no sólo abordarlo desde un punto netamente teórico sino también comprometerse con la realidad cotidiana y enigmática que nos caracteriza como sujetos y como suelo. De aquí que, pensar lo "latinoamericano", para les intelectuales y pensadores, no sea otra cosa que asumir totalmente los senderos misteriosos de Nuestra América. No se trata de desconocer la tradición filosófica previa, sino de asimilarla en función de los problemas de nuestro tiempo y nuestro lugar:

Claro está que no se trata de negar la filosofía occidental, pero sí de buscar un planteo más próximo a nuestra vida. Cuando Kant enuncia su teoría del conocimiento, lo hace porque en ese momento era imprescindible. Lo mismo ocurre con Hegel, quien expresa el sentir íntimo de la burguesía alemana de su tiempo. Descartes había pensado su cogito, ergo sum, porque así lo exigía el siglo de Richelieu con su razón de estado. El pensar europeo, como bien lo demostró Dilthey, siempre se vinculó a un estilo de vida (Kusch, 2007, pp. 263-264).

Este compromiso por parte de les filósofes que deciden reflexionar sobre lo latinoamericano se encuentra atravesado no sólo por una realidad aún desconocida en gran parte, sino también por la carencia de un marco conceptual que refleje los temas, cuestiones y/o problemas propios. Un "pensar propio" requiere un sólido arraigo 
cultural, es decir, el suelo como base de todo teorizar ya que no hay otra condición universal que la del estado-de-yecto en un suelo particular. El suelo o espacio físico es la base de todo arraigo y, por ello, de todo pensamiento que surge en el devenir de la temporalidad. Por este motivo, el sujeto sin conocimiento de su propio suelo no puede reflexionar ni tampoco generar cultura. Esta intersección entre pensamiento, cultura y suelo propio es un aporte sustancial en la obra de Kusch:

Se trata, antes bien, de captar libremente nuestra verdad sudamericana que, para nuestra mentalidad excesivamente esquemática de clase media intelectual, resulta desde todo punto de vista sorprendente e imprevista. Es preciso pensar que la comprensión de un 'sentido' de la vida latinoamericana debe rebasar las barreras que nosotros colocamos para ella (Kusch, 2007, pp. 271-272).

El problema que surge aquí radica en que la mayoría de les pensadores e intelectuales latinoamericanos manifiestan temor y rechazo de reconocerse como tales y, con ello, de pensar lo propio, lo latinoamericano. Tal temor y rechazo fue la causa principal y motora de aquella dicotomía arbitraria impuesta y manipulada dentro del pensamiento, es decir, la dicotomía pensamiento-culto y pensamiento-popular.

El pensar culto se rige por el modelo de la racionalidad científica y técnica, es sistemático y tiende a cristalizarse en forma enciclopédica. Pensar lo popular es, en cambio, pensar un área marginal de nuestras naciones y de nosotros mismos (Mareque, 1989, p. 54).

Por este motivo, ya en el Exordio ${ }^{1}$, brinda un gran adelanto del tema y la metodología que utilizará para alcanzar su objetivo. Así pues, en los primeros párrafos encontraremos la exposición el motor de su investigación: pensar y definir lo "americano". De aquí que, para alcanzar dicho objetivo implique que se realice un sondeo e investigación hacia las capas más profunda del ser y sentir latinoamericano, hacia las vivencias inconfesables. Es decir, esta tarea que emprenderá todo filósofo no requerirá de métodos y técnicas tradicionales o ya establecidas por la academia sino más bien con el reconocimiento de una verdad

\footnotetext{
${ }^{1}$ El Exordio, como parte estructural de la obra América Profunda, implica la intención de llamar la atención del lector y, a su vez, funciona como una "advertencia" sobre el contenido y la petición que realizará en el cuerpo del libro.
} 
Exploraciones en torno a los conceptos de Rodolfo Kusch, el pensamiento nacional y los levantamientos populares latinoamericanos

interior aún no contemplada ni asumida. Se debe ir hacia el hombre mismo, hacia el material empírico viviente y hacia las vivencias y/o experiencias. Esto implica,

Comer junto a su gente, participar de sus fiestas y sondear su pasado en los yacimientos arqueológicos, y también tomar en cuenta ese pensar natural que se recoge en las calles y en los barrios de la gran ciudad. Solo así se gana firmeza en la difícil tarea de asegurar un fundamento para pensar lo americano (Kusch, 2007, p. 5).

Asimismo, partiendo de lo dicho anteriormente, la verdad interior que se debería asumir como filósofos e intelectuales es el asumir nuestra condición como sujetos latinoamericanos con una historia particular y una identidad en construcción, es decir, de apropiarnos de aquella "continuidad del pasado americano en el presente [a construir]" (Kusch, 2007, p. 4). Esta gran tarea es una osada tarea que no todos los filósofos latinoamericanos estarán dispuestos a realizar, ya que supone salirse de los métodos y formalismos académicos tradicionales. Dejar de lado las estructuras filosóficas académicas no sólo implica perder seguridad al momento de teorizar algo sino también que "nos avergüenza llevar a cabo una actividad que requiere forzosamente una verdad interior y una constante confesión" (Kusch, 2007, p. 5).

Kusch no sólo interpela a les pensadores latinoamericanos, sino que también brinda algunos tópicos para realizar dicho camino hacia lo propio, asumiendo que se trata de un camino a medio hacer y desconocido aún. Entre estos tópicos conceptuales abordaremos los siguientes: la dicotomía ser-estar, el hedor y la pulcritud y, por último, la fagocitación.

La osadía de Kusch ha sido identificar dos elementos esenciales que caracterizan a dos culturas antagónicas que difieren tanto en el modo de concebir el mundo y las cosas como en el modo de existencia. Si bien tanto el sujeto occidental como el latinoamericano se encuentran arrojados (yectos) en un tiempo-espacio determinado, sus formas de solucionar ese estado existencial y vital son diferentes. El sujeto eurooccidental se caracteriza, principalmente, por abordar los males que lo afectan en su propio contexto a través de la modificación del mismo. Para ello, recurre a la creación de herramientas y elementos a través de la ciencia y la técnica con la finalidad de 
manipular y alterar la naturaleza hostil en función de su propio interés. Se puede decir que:

La cultura occidental, en cambio, es la del sujeto que afecta al mundo y lo modifica y es la enajenación a través de la acción, en el plano de una conciencia naturalista del día y la noche, o sea que es una solución que crea hacia afuera, como pura exterioridad, como invasión del mundo o agresión del mismo (Kusch, 2007, p. 112).

Este modo de proceder hacia afuera y modificar el entorno sólo puede darse en el marco de una concepción dinámica de la cultura. Es decir, la alteración de la naturaleza y/o contexto que realiza el sujeto euro-occidental termina creando otro mundo, otro contexto que se superpone sobre su propio contexto. Esta segunda naturaleza se caracteriza por estar construida a imagen y semejanza de las necesidades e inseguridades de ese sujeto, siendo organizado de forma tal que cada individuo tiene un rol y un objetivo que cumplir en él. No hay lugar para el caos, concebido como aquello que desestabiliza el orden y el progreso. Sin dudas, el hombre occidental es el sujeto del progreso y de la eliminación de los males que interrumpen la productividad. Así, como lo aclara Kusch:

El occidental, en cambio, encuentra verdades inestables porque suprime la ira divina y crea un mundo material como la ciudad, la cual imita la naturaleza [...] El occidental se aísla del mundo, porque ha creado otro, integrado por maquinarias y objetos, y que se superpone a la naturaleza (Kusch, 2007, p. 114).

Sin embargo, en las raíces profundas de Nuestra América, Kusch encuentra indicios de un modo de ser diferente, que se caracteriza por sentirse parte del todo $y$, por ello mismo, un integrante más de la naturaleza. Su sentir es el del estar, es decir, no busca alterar ni modificar su contexto sino más bien armonizar y contemplarlo. Su actividad respecto del mundo y la naturaleza es meramente contemplativa y, con ello, desarrolla una actitud introspectiva. Así pues, "El mundo del estar no supone una superación de la realidad, sino una conjuración de la misma. El sujeto continúa teniendo la realidad frente a sí, porque carece de ciencia para atacarla y también de agresión" (Kusch, 2007, p. 116).

El hombre latinoamericano ha forjado una cultura estática, que se limita a observar y desentrañar los misterios que esconden la naturaleza y el mundo. La cultura 
Exploraciones en torno a los conceptos de Rodolfo Kusch, el pensamiento nacional y los levantamientos populares latinoamericanos

estática es propia de las mesetas y las alturas -Kusch la encuentra en la cultura quechua- como el espacio de los acontecimientos mágicos de difícil comprensión a través de la razón. El mundo es aquí un locus propio e identitario donde intervienen tanto las fuerzas mágicas y naturales como la existencia efímera del hombre. Así expresa, a modo de síntesis:

Resumiendo, diremos que la cultura quichua es la consecuencia de una actitud estática, de un mero estar que se aferra a la meseta para perseguir el fruto. Y como solución espiritual de esa situación, se priva de un mundo azaroso mediante el ayuno, para encontrar en la intimidad el fundamento de su existencia. En esto último radica la sabiduría de la vieja América (Kusch, 2007, pp. 118-119).

La dicotomía arquetípica hedor/pulcritud expresa aquel drama existencial presente en las estructuras internas de la sociedad latinoamericana. Por un lado, contamos con la pulcritud propia de las sociedades modernas donde reina la civilización, la ciencia y el progreso. Aquí nos encontramos con figuras importantes como el ciudadano, el intelectual y las clases medias. Y por el otro, tenemos el hedor que es característico de todo lo periférico y lo excluido de las sociedades modernas. En este sector, encontramos a los individuos salvajes y peligrosos como el gaucho, el indio y lo popular. De ellos, sólo se espera el caos, la barbarie y el retraso de la vida en la intemperie.

El hedor es todo lo que representa el peligro interno, aquello que para el hombre occidental se debe erradicar o, incluso, cortar de raíz para evitar futuros brotes. Por este motivo, el hedor es lo que vive por fuera de la ciudad o en las zonas periféricas de la civilización.

El hedor es un signo que no logramos entender, pero que expresa, de nuestra parte, un sentimiento especial, un estado emocional de aversión irremediable, que en vano tratamos de disimular. Más aún, se trata de una emoción que sentimos no sólo en el Cuzco, sino frente a América, hasta el punto de que nos atrevemos a hablar de un hedor de América (KUSCH, 2007, p. 12).

También, se puede decir que lo que hiede es aquello que representa lo grotesco y feo que toda cultura quisiera ocultar, con el fin de construir una imagen pulcra y blanca hacia afuera. El hedor es la cara de la moneda que se trata de ocultar a cualquier 


\section{Pageau et al.}

costo, ya que lo que hiede siempre está ahí, oculto, pero manifestándose en los intersticios de la civilización.

El hedor de América es todo lo que se da más allá de nuestra populosa y cómoda ciudad natal. Es el camión lleno de indios, que debemos tomar para ir a cualquier parte del altiplano y lo es la segunda clase de algún tren y lo son las villas miserias, pobladas de correntinos, que circundan a Buenos Aires (Kusch, 2007, p. 12).

Ahora bien, la pulcritud es aquello que está presente en las sociedades modernas bajo el manto de la civilización, el orden y el progreso. La pulcritud es todo lo que les ciudadanes aspiran y creen posible y necesario. Las ciudades son habitadas por aquellas personas que son productivas y civilizadas que buscan "ser alguien" en este mundo. Para les ciudadanes, la ciudad es su hábitat y el estado-gobierno es su forma posible ya que sin gobierno sólo se vive en el caos. La pulcritud es aquella cara de la moneda que se quiere y se debe mostrar, ya que es la imagen de lo occidental y lo estéticamente aceptable. De aquí que, Kusch explica:

Y en el juicio aquel sobre el hedor de América y sobre la afanosa pulcritud, se halla implícito el afán de encubrir una ira que nadie quiere ver. Está en juego un planteo primario que el hombre siempre ha necesitado, pero que el caparazón de progresismo de nuestros ciudadanos e intelectuales -progresismo alimentado casi exclusivamente en la Europa burguesa del siglo XIX- trata de mantener a raya, porque, si no, ellos perderían salud y bienestar (Kusch, 2007, p. 18).

Por último, si el hedor representa un modo mítico y religioso de estar en el mundo donde el hombre se inmiscuye en el suelo, en el paisaje y con la naturaleza, es sin dudas la América profunda, la América enigmática e impenetrable hasta ahora. En cambio, la pulcritud es todo lo contrario, ya que es la expresión racional discriminadora y excluyente que cubre bajo el velo de la civilización donde el sujeto construye un nuevo mundo por fuera del suelo y el paisaje donde habita. De aquí que, el pensador argentino diga, que esta cultura es de lo superficial.

La fagocitación es un concepto peculiar ya que el mismo procede de la Biología y, específicamente, del verbo fagocitar. Fagocitación es el proceso mediante el cual un organismo incorpora, asimila y digiere aquellos elementos extraños para hacerlos propios sin destruirlo. Se puede decir que este proceso está relacionado con el instinto 
Exploraciones en torno a los conceptos de Rodolfo Kusch, el pensamiento nacional y los levantamientos populares latinoamericanos

de supervivencia que todo organismo atraviesa durante su existencia. Kusch, como pensador latinoamericano, manifestó una actitud integradora respecto de lo Occidental ya que su objetivo siempre fue definir y tematizar lo latinoamericano. De aquí que, la fagocitación sea tomada desde la acción curativa presente en ella, es decir, Latinoamérica necesita incorporar lo Occidental, asimilarlo y hacerlo propio para poder expresar y estructurar lo propio.

Sin embargo, se debe aclarar que la fagocitación es una actividad implícita, es decir, una actividad que se da por abajo o en el interior de la cultura del sujeto que, normalmente, no se ve, pero se la puede sentir y experimentar en el lenguaje y en la subjetividad de los individuos. Por este motivo, "la fagocitación no es consciente sino que opera más bien en la inconsciencia social, al margen de lo que oficialmente se piensa de la cultura y de la civilización" (Kusch, 2007, p. 197). De aquí que, el pensador argentino comente, que por más que en Latinoamérica las ciudades se muestran civilizadas y pulcras, eso no implica que se hayan cortado las raíces del suelo o que se haya erradicado del todo el hedor.

Ahora bien, la fagocitación es también catalizadora de todos los opuestos que operan en una cultura. Así, desde una clara influencia hegeliana, la fagocitación permite realizar aquel proceso de síntesis donde se superan los opuestos a través de la reconciliación y la aparición de algo nuevo para explicar lo propio. La fagocitación es una propuesta de salida a los antagonismos at infinitum dentro de la cultura. Tal salida es la capacidad sanadora y re-creadora de aquellos elementos extraños y extranjeros. El sentido de esta capacidad radica en encontrar el equilibrio entre lo extraño y lo propio para alcanzar aquella sabiduría donde convive el caos y el orden hasta transformarlo en algo propio y útil culturalmente.

\section{Miradas sobre la propuesta emancipatoria de J.J. Hernández Arregui desde Kusch}

Contemporáneo de Kusch, también formado en el campo académico modernoeurocentrado- de la filosofía, el gran intelectual J.J. Hernández Arregui (1913-1974) ha sido quién definió y organizó una reflexión crítica de carácter emancipatorio, dentro de una línea ideológica que él caracterizó como "izquierda nacional”, una reflexión de 
carácter nacional y popular, que partía de las falencias tanto de la derecha como de la izquierda clásicas, ambas interpretadas como distantes o separadas de los intereses, necesidades y demandas históricas del pueblo argentino ${ }^{2}$. Su obra ensayística y su praxis militante, responsable del acercamiento de la juventud de la época al peronismo-el gran movimiento de liberación popular, según el pensador-, desvela el carácter colonizante y eurocentrado de la cultura oficial, la de las oligarquías y burguesías agro-industriales, como su actitud íntimamente extranjerizante. Su prédica antiimperialista se alimenta en un revisionismo histórico inspirado, especialmente, en FORJA, Jauretche y Scalabrini Ortiz, en una zona del campo intelectual que se conformó como una periferia del pensamiento argentino de la época ${ }^{3}$ y que encontraba un eco en las luchas de liberación del surcontinente-Cuba, Chile, Bolivia u otros- $y$ en el resto del entonces llamado Tercer Mundo, el mundo alternativo deseado por los condenados de la tierra.

Reconocemos en su gesto vital (en su oficio de docente, ensayista, difusor, militante), el actuar de un gran descolonizador, dentro de los límites que permitían los condicionamientos epocales, donde el marxismo heterodoxo era la herramienta conceptual hegemónica, abocado a la construcción de una nación autónoma y soberana, que sería insertada de modo diferencial en el capitalismo mundial, en un proyecto que $\mathrm{H}$. Arregui quiso pensar como la antesala a la construcción de un auténtico socialismo latinoamericano (Peronismo y Socialismo, 1972). Dentro de las contradicciones propias, también ${ }^{4}$. Subrayamos la naturaleza multidisciplinaria y el carácter muy riguroso y ampliamente documentado de sus escritos, tomando desde la

\footnotetext{
2 Es muy conocida e imprescindible la labor de divulgación de Norberto Galasso (2012), y resultan interesantes los aportes de Martin Gerlo (2014), en cuanto a los aspectos principales del pensamiento arreguiano.

3 Torres Roggero caracteriza a Jauretche como profeta de la decolonialidad, en oposición a los profetas del odio, es decir, los intelectuales colonizados y eurocentrados, la "intelligentzia" -decía Jauretche-, con su actitud que facilita la dependencia del país (Pensamiento Argentino, Ed. del Signo, 2010, p.49-85.) Observa que Jauretche y Scalabrini Ortiz, reconocían "a las masas populares como productoras de saber" (Torres Roggero, 2010, p. 55)

4 Jessica Jones (2010, p.153) estima que el intelectual progresista, en contexto de colonialidad, se encuentra "obligado a celebrar su otro para definirse en conjunto como una nación unida en contra de la influencia extranjera".
} 
Exploraciones en torno a los conceptos de Rodolfo Kusch, el pensamiento nacional y los levantamientos populares latinoamericanos

economía política hasta la literatura y la cultura en sentido amplio, en una mirada histórica y descolonizante.

Puede parecer sorprendente poner en dialogo dos voces aparentemente tan distintas como las de H. Arregui y Kusch. Pero, justamente, el diálogo verdadero, ¿no es acaso el que permite la escucha de quien opina de modo diferente u opuesto al de uno? Entendemos que ambos reflexionan y actúan buscando la manera de comprender la diversidad americana, la naturaleza de su alma, el sentido de su dolorosa experiencia histórica, y ambos piensan siendo sensibles a la desposesión, sensibles a la herida colonial (Mignolo, 2007) que sigue sufriendo un pueblo que ha sido grande.

Así, entusiasmados y sintiéndonos interpelados por ambas propuestas críticas, nos propusimos reflexionar acerca de algunas zonas de la trayectoria y pensamiento de H. Arregui, partiendo de ideas de Kusch, las cuales, por cierto, son portadoras de un valor claramente decolonial, de desprendimiento de los paradigmas y categorías modernas, como las que presentamos más arriba. Queremos, sencillamente, ensayar otros puntos de entradas, suscitar otras miradas a este pensamiento portador de esperanza en pos de la liberación popular.

De entrada, nos interesa rescatar una anécdota, un evento que surgió en el lugar de Resistencia, nuestra capital chaqueña, desde la periferia resistente, desde donde otros filósofos o militantes también se expresaron y actuaron ${ }^{5}$. Nos sirva para ver que H. Arregui se coloca del lado del supuesto hedor americano, ante la pulcritud moderna, ficticia aunque reivindicada, y desdeñosa del pueblo. Demuestra también su necesidad de poner a prueba sus ideas, dialogar con los estudiantes, en la diversidad de posicionamientos ideológicos o políticos.

Decía H. Arregui, en el prólogo de un libro suyo, que acababa de ser publicado (1963, Hachea):

\footnotetext{
${ }^{5}$ En su texto del 2008, una mirada retrospectiva de su recorrido intelectual y militante, Dussel subraya la vigencia que han mantenido algunos aspectos que se había planteado en la UNNE, Resistencia, cuando dictó un curso en el departamento de Filosofía, en 1966, y donde publicó varios trabajos.
} 
El bosquejo del trabajo fue una conferencia leída en 1961 con el mismo nombre: ¿Qué es el ser nacional?, bajo los auspicios del Movimiento de Estudiantes Reformistas de la Universidad Nacional del Nordeste, en la ciudad de Resistencia. Un público heterogéneo y atento, con presencia de obreros, estudiantes y diversos grupos ideológicos, fue el primer indicio de que el tema interesaba. (subrayado nuestro) $(1973$, p.7).

Y prosigue el autor expresando su gratitud por otra reacción a su conferencia en una provincia "profundamente argentina"-Santiago del Estero- donde estaban representadas las más diversas tendencias,

(...) cosa difícil de lograr en provincias chicas, peronistas, radicales, nacionalistas, gente de izquierda - recibió mis palabras con tal fervor nacional a pesar de mis ideas en tantos sentidos consideradas extremas, que no puedo menos de recordar a la distancia con gratitud la adhesión de ese público provinciano. Y una vez más confirmé mi certeza de que el país verdadero está en las provincias más humildes (subrayado nuestro) $(1973$, p. 8).

Observamos, pues, que $\mathrm{H}$. Arregui no busca predicar a los convertidos ni convencer a otros intelectuales del campo adverso, sino que se dirige al pueblo en su diversidad, lo dice él, reconoce y valora la heterogeneidad de las varias proveniencias. Se expresa y reflexiona confiado en hallar alguna verdad del lado de los más humildes, algo cercano a la idea de Dussel del pueblo como bloque social de los oprimidos (Dussel 2006, 20 tesis de política). Así, no puede ser más claro su posicionamiento del lado del hedor, del pueblo hondo, el cual se concreta también en sus planteos críticos, por ejemplo al retomar y desarrollar los conceptos de colonización pedagógica, o colonialismo interno al cual está sumiso tanto el pueblo como la burguesía, fenómenos que causan, según él, la despolitización del pueblo y lo hace ciego a las caminos de su liberación. Lo veremos más adelante, aunque sea de modo panorámico.

En dicho prólogo, H. Arregui citando a otro lugar de cálida recepción, la ciudad de Santa Fe, 1962, muestra, y nos parece esencial, su voz sentipensante, al expresar que "quizá, en los momentos críticos de un país, los únicos libros objetivos son aquellos escritos con la sangre caliente y la mente fría que los hace neutros a toda pasión innoble". Del mismo modo, en la advertencia a la segunda edición (1964) de 
Exploraciones en torno a los conceptos de Rodolfo Kusch, el pensamiento nacional y los levantamientos populares latinoamericanos

Imperialismo y Cultura (1957), confiesa que inició el libro luego de la caída del General Perón, por vía del golpe oligárquico militar del 1955. A raíz de la revolución del General Juan José Valle, fue encarcelado y presenció la tortura de obreros y de una joven mujer, evocando, de modo retrospectivo,-“esas cosas no se olvidan"- el odio profundo que acompañó la escritura y posterior mirada a este libro, odio inspirado en la materia tratada: la traición al pueblo, los intelectuales colonizados. "Más aún, no era odio lo que lo inspiraba, sino el amor al país" (1957, p.7). De modo paralelo, entendemos que en América Profunda, Kusch no se aparta de su subjetividad, al confesar sus propias impresiones de turista blanco y moderno en Cuzco, conceptualizando luego hedor y pulcritud, para "sondear las vivencias (...) en los rincones oscuros del alma" y otorgando un lugar importante a su intuición en la elaboración conceptual filosófica (Kusch, 2007, p. 4). Es en este sentido que el prologuista-Norberto Maicas (A.P., ed. Biblos, 1999, p.9)- retoma palabras de Kusch: "existe una especie de obsesión por la racionalidad, que no permite ver cualquier otra posibilidad".

Reflexionando, H. Arregui lo hace de modo situado, y todavía en este libro acerca del ser nacional, da cuenta de su sensibilidad al pensar del pueblo, cuando entiende que esta recepción popular e inesperada de un filósofo- calificado en su momento de "intelectual maldito" (fue expulsado de la universidad y fue recorriendo de los márgenes profesionales y geográficos, escribiendo ensayos y presentando conferencias en las provincias) - "explica el cambio de la conciencia histórica de la América Hispánica frente al imperialismo". Como lo remarcaba en 1957, "el dilema es de hierro: o Nación o Imperialismo" al finalizar el Prefacio a la primera edición de Imperialismo y Cultura (2005, p.22)

En una época marcada por el llamado "trasvasamiento generacional" dentro del peronismo especialmente, el intelectual se sitúa del lado de los jóvenes, educados por padres que han padecido la colonización pedagógica, y ubica en la juventud de izquierda la que asiste a la nacionalización ideológica, es decir, el despertar de un nuevo sentimiento de pertenecía, crítico de la historia falseada: "es falsa la historia que nos enseñaron", declara H. Arregui, en Formación de la conciencia nacional, del 
1960 (2011a, p. 257). Este hecho de la vitalidad de la izquierda, dice él, no se debe negar ni producir temor, y lo leemos, retrospectivamente, como un momento de posible o parcial desprendimiento de esa juventud. Un modo de sana toma de conciencia histórico-política en tiempos de censuras desde el Estado mismo.

Resulta obvio en estos aspectos la toma de partido en contra de la pulcritud de una elite tanto hipócrita como dañina, y entonces, a favor de lo que son descalificados con criterios superficiales, desde el dicho hedor. Ahora, veamos cómo podríamos reflexionar desde las ideas de ser y estar.

Ser, nos explica Kusch, es un Ilegar-a-ser-moderno, el cual implica una trayectoria de vida, de cultura, de historia que siguiera las vías, moldes y modelos ostentados por los modernos. Nos parece que la pregunta, la problemática por la comprensión del ser nacional, planteada por Hernández Arregui, si bien no se aleja del todo de este ser, no expulsa la dimensiones del estar. Indagamos un poco.

Desde su búsqueda de comprensión de la realidad de la nación en contexto de dependencia económica y cultural, dirigido desde Inglaterra o EE. UU y facilitado por la penetración pedagógica permitida desde las élites, la definición de una persona nacional sino un proyecto de ciudadanía aparece como urgente para el H. Arregui. Luego de tantear algunas pistas, a continuación, aporta una definición más completa, algo que citamos, extensamente:

El "ser nacional" emerge ahora, (...) no como paz, sino como guerra. (...), en última instancia, pugna por cimentarse sobre las oposiciones de las clases sociales que luchan por el poder político. En síntesis, el "ser nacional" no es uno sino múltiple (1973, p.19).

(...) Como dijera Manuel Ugarte, "unos pueblos viven en mayúscula y otros mueren en minúscula". De acuerdo a la categoría a que se pertenezca, el "ser nacional", la patria, la comunidad nacional, la cultura nacional, a través de las clases sociales en tensión, tiende a refractarse de modo distinto en un país dominante que en un país dominado $(1973$, p.20).

(...) en una comunidad establecida en un ámbito geográfico y económico, jurídicamente organizada en nación, unida por una misma lengua, un pasado común, 
Exploraciones en torno a los conceptos de Rodolfo Kusch, el pensamiento nacional y los levantamientos populares latinoamericanos

instituciones históricas, creencias y tradiciones también comunes conservadas en la memoria del pueblo, y amuralladas, tales representaciones colectivas, en sus clases no ligadas al imperialismo, en una actitud de defensa ante embates internos y externos, que en tanto disposición revolucionaria de las masas oprimidas se manifiesta como conciencia antiimperialista, como voluntad nacional de destino (1973, p.22).

Observamos inicialmente la "necesaria" oposición de clase, de la interpretación marxista-una dicotomía que Kusch supera sin ignorar-, y la idea de que en el plano sensible, como dice el autor, la idea más amplia y expresiva, pero no definida, sería la de patria. En el plano político, cuando llega a plantear la comunidad de lengua, de pasado, instituciones, creencias y tradiciones, es difícil no pensar en la propuesta de B. Anderson de "comunidad imaginada" ${ }^{6}$. Este acercamiento se debe problematizar pues como expresa H. Arregui, existe la diferencialidad de un país dominado, neocolonizado, donde la percepción que se tiene del país y su historia está condicionada por la colonización pedagógica, por el colonialismo interno, fenómenos ampliamente discutidos e ilustrados por el autor en buena parte de su obra ensayística. Y es justamente por ello que llega a presentar el concepto de ser nacional como una cuestión programática, cuando dice, "disposición revolucionaria de las masas oprimidas se manifiesta como conciencia antiimperialista, como voluntad nacional de destino". Sintetizando desde Kusch, se trata de un llegar-a-ser, pero en plenitud, una conciencia que parte de un estar o saber estar en contexto de colonialismo interno o de imperialismo. El ser nacional se definirá por y en su cultura de lucha por la liberación nacional, una liberación que de hecho, por pasar por las mismas categorías modernas del Estado Nación, queda probablemente más del lado de la emancipación iluminista.

Este ser nacional se identifica con el pueblo, claramente, y se encuentra caracterizado por la comprensión que tiene u ofrece el intelectual acerca de la cultura,

\footnotetext{
${ }^{6}$ A diferencia de lo propuesto por Ferrari (2003), desde nuestra comprensión, la cuestión de pertenencia a la Nación, especialmente en nuestras dolorosas Repúblicas, es íntimamente vinculada a la experiencia compartida de las continuas luchas históricas de liberación popular, sin expulsar del todo el componente "imaginado", sino subsumiéndolo.
} 
en términos amplios. Más allá de la cuestión de falseamiento cultural típico de la década del treinta, y su retorno en el 1955- los intelectuales y las élites, especialmente, vistas como "fotocopia espiritual de Europa" (Imperialismo y Cultura)- cuando piensa la cultura y la historia, H. Arregui plantea la necesidad de partir desde la conquista, desde la dualidad americana-hispana, y no se olvida ni de los indígenas ni de los afrodescendientes. Por prueba tenemos esta reflexión: "Hay, pues, que retroceder a España, y al hecho de la conquista, calar en las culturas indígenas y en el período hispánico," que desarrolla en algunos apartados sucesivos (1973, p. 27).

Además, sus comentarios acerca de cultura en Nacionalismo y Liberación (1969) atestiguan una valiosa apertura/inclusión, más allá del proletariado urbano del marxismo ortodoxo. Por ejemplo, cuando observa fenómenos de transculturación de formas culturales regionales, o cuando presenta y comenta citas de poesías en lenguas quechua y guaraní, junto con su traducción al castellano. El intelectual identifica además en la cultura popular sus raíces española, indígena y "sin excluir el importante elemento negro" (2004: 158). Es decir, no encierra tanto la noción de cultura argentina en el único componente criollo, como se suele pensar. Tampoco el folclore está contemplado de forma reduccionista, sino como "saber popular, lo que el pueblo sabe tal como lo sabe (...) lo que hace todos los días" y se lo asocia a "una forma políticamente combativa", especialmente en el interior". Además, observa que "el intercambio entre la ciudad y el campo modifica tanto al tipo urbano como al rural" (2004: 163), lo cual demuestra un modo más complejo de pensar la usual dicotomía centro/periferia. Quizás podamos llegar a hablar de estar-siendo en el modo que tiene H. Arregui de pensar, de modo sensible y situado, la problemática del ser nacional, quizás al modo de un estar-haciendo kuscheano.

De todos modos, como vimos, H. Arregui reivindica claramente al pueblo como sujeto histórico; muy lejos de él una actitud urbana y colonial, descrita por Kusch, donde "la categoría básica de nuestros buenos ciudadanos consiste en pensar que lo que nos es ciudad, ni prócer, ni pulcritud no es más que hedor susceptible de ser 
Exploraciones en torno a los conceptos de Rodolfo Kusch, el pensamiento nacional y los levantamientos populares latinoamericanos

exterminado" (Kusch, 2007, p.13) como escribe en la introducción a América profunda.

Encontramos en algunos aspectos del pensamiento de $\mathrm{H}$. Arregui otras señales de su pensar desde el estar-siendo americano, revelador del actuar profundo del colonialismo interno, especialmente cuando explica, en Nacionalismo y Liberación:

Ya se ha dicho que el colonialismo es mucho más que un fenómeno económico (...) Es un complejo mecanismo de vías entrecruzadas e invisibles, enderezado arteramente a la deformación, invalidez y empastelamiento mental de las clases colonizadas. Más aún, la muralla psíquica opuesta a la liberación es esa, no siempre consciente, mentalidad colonial. La colonización abarca todas las esferas, materiales y espirituales, del país sometido (2004, p.136).

Aquí, el fragmento estudiado asocia primero la condición (neo)colonial a un sistema orgánico que actuaría de manera compleja y subrepticia, una agencia que supera -sin por lo tanto obviar- el aspecto económico. Este "mecanismo" se asimila a un conjunto coherente de fuerzas que actuarían al modo de una lógica de poder que violenta la integridad del espíritu del pueblo. Tal mecanismo impide una toma de conciencia y una concepción clara de la realidad, puesto que actúa como conciencia sobre-impuesta, como un molde predeterminado y censor de toda originalidad. Y es en este sentido justamente que la colonialidad del poder abarca todas las esferas de la experiencia humana, aquí resumida por $\mathrm{H}$. Arregui a "todas las esferas materiales y espirituales". No será acaso este mecanismo complejo, esta muralla psíquica propios justamente de esta pulcritud, que necesita callar lo propio profundo, tapar el hedor, por ser visto como vergonzoso, y se desalma por sus pretensiones de grandeza, en aspiraciones a ser blanco moderno en América, a ser, en definitivo, lo que no son.

Entendemos que lo que él busca conceptualizar puede asociarse directamente a la acción de la colonialidad del poder (Quijano 1992) que implica las del saber y del ser, pues el conjunto de estas fuerzas atenta tanto contra la persona como contra su modo de percibir y explicar el mundo. 


\section{Pageau et al.}

Este espíritu de pulcritud es también el que habita las élites y burguesía coloniales en América, especialmente en el siglo XIX, en contexto de endurecimiento del régimen colonial (consecuencia del absolutismo de Fernando VII) esos criollos que aspiran desesperadamente a la ascensión económica y social, aquellos que anhelan llegar-a-ser, poco importa su estar, de un modo que tiene resonancia hasta la actualidad, en los estragos que produce el neoliberalismo. Dice, al respecto, Hernández Arregui, en Formación de la conciencia nacional:

Desde ya debemos señalar -y el hecho es de vital importancia- que aquí en América Hispánica el liberalismo penetró más que como ideología progresista como reflejo residual de la evolución liberal europea (...) en tanto ideología de la Europa colonizadora, un medio de opresión y dominio envasado tras el rótulo de libertad, democracia, progreso, derechos humanos, etc. (2011a, p.25).

$Y$ añade:

Así, si bien el liberalismo en sus orígenes fue negación y superación del orden social del feudalismo, a fines del siglo XIX el liberalismo mercantil se transforma en imperialismo. De modo específico, el siglo XIX asistió "junto con el desarrollo de la técnica -en sí misma la más grande conquista humana- a la subversión cultural de un mundo que revolucionó (...) toda la existencia social del hombre (2011ạ, p. 38).

Es decir, la importación/imposición de esquemas de pensamientos de origen iluminista ha significado, a fin de cuentas, una traición al humanismo (Césaire, Discurso sobre el colonialismo, 1950), y no ha permitido concretar cabalmente una modernidad plenamente emancipatoria, a pesar de las promesas. Todo lo contrario. El bienestar o mejor dicho, el mejor-estar imaginado, el llegar-a-ser moderno, blanco, capitalista, pulcro, ha sido el privilegio de pocos, y en contraste, para la mayoría en la América profunda, ello ha significado la experiencia de la enajenación, la exclusión o la opresión. Exit, entonces el estar-en-sí en su entorno y en la heterogeneidad de las relaciones intersubjetivas, en la diversidad cultural; bienvenido el llegar a ser otro, pretender ser lo que no somos, y así llegar a ser seres escindidos y separados.

Kusch explica que para ilustrar cómo funciona el hedor, "haría falta algo como la extracción de un tumor, como una revelación, romper el caparazón de progresismo 
Exploraciones en torno a los conceptos de Rodolfo Kusch, el pensamiento nacional y los levantamientos populares latinoamericanos

de nuestro ciudadano, su mito inveterado de pulcritud, ese fácil montaje de la vida sobre cosas exteriores, como ciudad, policía y próceres" (Kusch, 2007, pp. 13-14) Sugerimos que esta extracción- sino de todo el tumor, seguramente una buena parte-, esta revelación dolorosa y violenta la está operando H. Arregui mediante el desvelamiento de la falsedad tanto de la pulcritud como del hedor, cuando logra pensar desde el estar-siendo de la América profunda.

\section{La descolonización de la mirada política a partir de Kusch}

Esta sección se encuentra guiada por una intuición y una pregunta ¿Las categorías forjadas por Rodolfo Kusch para pensar la especificidad ontológica de América pueden ser "puestas en práctica" para descolonizar la mirada sobre los movimientos políticos de carácter insurreccional ocurridos en nuestro continente durante la historia reciente?

Frente a marcos teóricos importados de manera acrítica, como la teoría de la acción colectiva o la de los nuevos movimientos sociales, que reproducen una mirada colonizada sobre la política latinoamericana (Galafassi, 2006) resulta indispensable recurrir a otras tradiciones gestadas en diálogo con los procesos de lucha de los pueblos.

Desde fines del siglo XX, y sobre todo en las primeras décadas del siglo XXI, se vienen produciendo diversos levantamientos populares en varios países de América Latina contra los efectos de la implementación de medidas económicas neoliberales. Puede hablarse de un primer "ciclo de movilizaciones" que tuvo como antecedente directo el alzamiento zapatista en México (1994) y se expresó principalmente en las geografías de Ecuador (1997 y 2000), Bolivia (2000, 2003 y 2005) y Argentina (2001). En todos los casos, los levantamientos populares tuvieron una serie de características particulares: lograron articular una diversidad de resistencias puntuales previamente construidas, carecían de una vanguardia o dirigencia centralizada y fueron capaces de generar rupturas institucionales (tanto en los organismos estatales como en las formas organizativas populares más formales), provocando una crisis de legitimidad de la 
hegemonía neoliberal en la región y dando lugar a varios "gobiernos progresistas" que asumieron parcialmente las demandas puestas en agenda por los movimientos sociales (Sader, 2008, p.73).

Posteriormente, y luego de una suerte de "restauración neoliberal" por parte de los gobiernos que precedieron a aquellos "progresistas", parece haberse desarrollado un segundo ciclo de movilizaciones en distintos puntos del continente. Levantamientos populares similares a los ocurridos a principios de siglo se sucedieron en Puerto Rico, Chile, Ecuador, Colombia durante 2019, en Haiti y Perú durante 2020, así como en Paraguay al momento de escribir este artículo. Los alcances de tales movimientos aún se encuentran en proceso de definición.

En otro escrito habíamos planteado la posibilidad de pensar a la luz de las categorías kuscheanas de estar y estar siendo los procesos de movilización de la sociedad boliviana durante el primer ciclo mencionado (Roman, 2009). Sin embargo, consideramos ahora posible proyectar esas reflexiones hacia un conjunto más amplio de fenómenos sociales.

El punto de partida común a todos los casos es la construcción de comunidad, en el sentido de una lógica que regula la vida cotidiana estableciendo relaciones sociales de solidaridad y apoyo mutuo, frente a la dinámica del "sálvese quien pueda" de la sociedad capitalista (Zibecchi, 2006). No se trata de un oasis en medio del individualismo y la competencia, sino de la creación permanente de nuevas relaciones sociales guiadas por una lógica diferente. Una especie de sustrato existencial al cual se vuelve constantemente para buscar soluciones a los problemas comunes.

Es posible pensar la lógica comunitaria como la recreación de un modo de existencia característico de la América Profunda. Ese estar o mero estar que asume la precariedad de la vida humana y la necesidad de asegurarla mediante la recreación constante de la comunidad, entendida como un fundamento no esencial, sino contingente. En la sabiduría popular, toda construcción es concebida como circunstancial y toda definición como transitoria, histórica y cambiante. 
Exploraciones en torno a los conceptos de Rodolfo Kusch, el pensamiento nacional y los levantamientos populares latinoamericanos

La idea central de esta organización consistía en una especie de economía de amparo, por oponerla a nuestras formas económicas, las que a su vez, desde el ángulo indígena, se pueden calificar como de desamparo... Una estructura así suponía un trasfondo angustioso que, sin embargo, no podía resolverse con la acción sino mediante una fuerte identificación con el ambiente (Kusch, 2007, p. 109)

A diferencia de lo anterior, la lógica occidental del ser o ser alguien, en su búsqueda de un fundamento último y permanente capaz de ser aprehendido en su totalidad mediante la razón humana, construye un universo artificial de objetos. Una segunda naturaleza bajo aparente dominio humano para resguardarse de la imprevisibilidad de la naturaleza y sus fuerzas irracionales, la "ira de Dios". De este modo, los individuos pierden sus lazos comunitarios y su supervivencia queda a merced de la ley de oferta/demanda.

Con todo esto, el hombre pierde la prolongación umbilical con la piedra y el árbol. Ha creado algo que suple el árbol, pero no es árbol. Como simple sujeto lógico que examina objetos y los crea, quiere ser un hombre puro, pero no es más que medio hombre porque ha perdido su raíz vital y, entonces, suple la ira de dios por su propia ira (Kusch, 2007, p. 130).

No obstante, desde el momento mismo de la invasión europea, en América interactúan el ser y el estar siguiendo una dinámica propia que Kusch denominó fagocitación. Si bien el ser occidental aparentemente se impuso en todos los ámbitos de la vida, el estar permanecería como un sustrato potencialmente capaz de asimilarlo y hacerlo coherente con su propia lógica. Así lo expresa la cultura popular, tanto urbana como rural, por su arraigo territorial y su cohesión interna. Mientras en sus manifestaciones más visibles las sociedades americanas parecen adaptarse al modo de vida occidental, las prácticas cotidianas tejen relaciones sociales que remiten a otro modo de vida comunitario. Allí, el fundamento de la vida no es la construcción de un dominio del hombre sobre la naturaleza para asegurar la supervivencia, sino la conjuración de la muerte mediante el fortalecimiento de la comunidad.

Pero esta misma oposición, en vez de parecer trágica, tiene una salida y es la que posibilita una interacción dramática, como una especie de dialéctica, es que 
llamaremos más adelante fagocitación. Se trata de la absorción de las pulcras cosas de Occidente por las cosas de América, como a modo de equilibrio o reintegración de lo humano en estas tierras (Kusch, 1976, p. 29)

La construcción de un entramado comunitario regido por la lógica del estar parece desplegarse y hacerse visible durante los momentos de irrupción política de las masas, desbordando a las dirigencias que hasta hace poco antes marcaban su camino y socavando las bases de aparatos institucionales que parecían inmutables. En esos momentos de estar siendo, de una "potencia que se manifiesta súbitamente para dar todo de sí misma" (Kusch, 1978, p. 10), la comunidad no construye cuerpos separados para la toma de decisiones ni para la lucha. Esto, a su vez, contribuye a su efectividad: la conformación de entidades autónomas capaces de desarrollar la totalidad de las funciones necesarias para su subsistencia, frente a un aparato represivo centralizado, especializado y con funciones únicas, incapaz de contener el desborde insurreccional.

En definitiva, estar siendo no implica la desaparición del ser, sino su germinación a partir de un arraigo comunitario, el estar. De este modo, se hace posible recuperar la autonomía del modo de ser americano porque el ser dejaría de ser una imposición extranjera y podría cobrar sentido en el marco de la comunidad en lucha.

Finalmente, cabe señalar que hay quienes observan las mismas características señaladas en determinados tipos de organización popular, como el piquete o las asambleas barriales. En el primer caso, el piquete constituiría una expresión del sustrato popular americano, tanto en lo cultural como en lo político. El corte de ruta y la organización territorial como constituyentes del arraigo comunitario del estar, y la identidad piquetera como concreción del estar siendo (Emiliozzi, 2007). En el segundo caso, aunque no hay una referencia conceptual directa a los conceptos de Kusch, se trataría de ámbitos donde es posible la generación de relaciones sociales asentadas en la lógica comunitaria, como base para la organización de la movilización popular (Colectivo Situaciones, 2002, pp. 174-175).

\section{Reflexiones finales}


Exploraciones en torno a los conceptos de Rodolfo Kusch, el pensamiento nacional y los levantamientos populares latinoamericanos

En primer lugar, las categorías sentipensantes desarrolladas por Kusch si bien se presentan a modo de oposiciones (ser/estar, hedor/pulcritud) en realidad cuestionan ideas recibidas y dominantes acerca de los modos de entender la naturaleza profunda del alma suramericano, y sus experiencias culturales históricas. Basado en estas pares de categorías enfrentadas y dialogantes, el fenómeno de fagocitación se organiza y de hecho desplaza la noción hegemónica y colonizante de la época, la anglófona "aculturation". Recordamos brevemente que en el campo de la antropología, la contestación de esta noción nos viene de la reflexión situada del antropólogo Fernando Ortiz, en 1940, en su famoso Contrapunteo cubano del tabaco y del azúcar, con el neologismo "transculturación", que permite profundizar, de modo paralelo a Kusch, la bilateridad de los intercambios culturales, pero en clave de colonialismo y capitalismo internacional. En segundo lugar, entendemos, desde las propuestas del nacionalismo popular de H. Arregui, que la reflexión sobre la cultura y la historia de una nación, aquí Argentina, no se puede desprender de las experiencias históricas concretas de liberación popular. Resultó interesante ver como la actitud de H. Arregui, entre otros, permite un filosofar atento justamente al estar, al pueblo en su hedor impuesto, su marginalización, pero en lucha contra la pulcritud de las élites y el sistema cultural eurocentrado y colonizante. El gesto descolonizante de H. Arregui, permite desvelar, por lo menos en parte, el modo en que se instala un pensamiento y una cultura que opaca la mente de la gente y lo despolitiza. Entendemos que muestra en parte cómo funciona esa pulcritud, este lado oscuro de la América. Finalmente, pensando en los movimientos políticos recientes en varios lugares del surcontinente, entendemos desde Kusch que un modo de descolonizar la mirada a estos sería contemplando la comunalidad inherente a las luchas populares. Para ello, puede resultar analíticamente prolífico comenzar a indagarlas desde un sistema categorial elaborado en diálogo con las culturas ancestrales americanas, pero también con las manifestaciones más sentidas de la cultura popular. De este modo, se abren nuevas sendas que podrían ser recorridas a la par por intelectuales y militantes, con la liberación como horizonte posible. 


\section{Bibliografía}

Colectivo Situaciones (2002). 19 y 20. Apuntes para el nuevo protagonismo social. Buenos Aires: De mano en mano.

Dussel, Enrique. (2006). 20 Tesis de política. Buenos Aires: Siglo Veintiuno Editores.

Dussel, Enrique. (2004). Transmodernidad e interculturalidad, Interpretación desde la filosofía de la liberación. Erasmus, Año V, Núm. 1/2, Córdoba, pp. 65-102.

Emiliozzi, M. (2007). La dimensión existencial del piquete. Rosario: CEFAL.

Ferrari, Jorge Luis, (2003). El pasado de una ilusión, la reconstrucción del ser nacional en Hernández Arregui, Revista de Historia Americana y Argentina, № 40, 2003, U. N. de Cuyo.

Galafassi, G. (2006). Cuando el árbol no deja ver el bosque. Neofuncionalismo y posmodernidad en los estudios sobre movimientos sociales. Revista Theomai, 14, 37-58.

Gerlo, Martín (2015). Marxismo y cuestión nacional: aportes de Hernández Arregui al pensamiento de izquierda. In XI Jornadas de Sociología. Facultad de Ciencias Sociales, Universidad de Buenos Aires.

Hernández Arregui, Juan José. (1973). ¿Qué es el ser nacional? Buenos Aires: Editorial PlusUltra. [1963]

Hernández Arregui, Juan José. (2004). Nacionalismo y liberación. Buenos Aires: Continente. [1969]

Hernández Arregui, Juan José. (2005). Imperialismo y cultura. Buenos Aires: Continente. [1957]

Hernández Arregui, Juan José. (2011a). La formación de la conciencia nacional. Buenos Aires: Continente. [1960] 
Exploraciones en torno a los conceptos de Rodolfo Kusch, el pensamiento nacional y los levantamientos populares latinoamericanos

Hernández Arregui, Juan José. (2011b). Peronismo y socialismo. Buenos Aires: Continente. [1972]

Jones, Jessica (2010). El espectro descolonial de la izquierda argentina, 1955-1976. En Zulma Palermo (comp.). Pensamiento argentino y opción decolonial. 149-182. Buenos Aires: Ed. Del Signo, Colección El Desprendimiento.

Kusch, Rodolfo (2007). América Profunda, Rosario: Fundación Ross - Tomo II [2007]

Kusch, R. (1978). Esbozo de una Antropología Filosófica americana. Buenos Aires: Castañeda.

Kusch, R. (1976). Geocultura del hombre americano. Buenos Aires: Fernando García Cambeiro.

Mignolo, Walter D. (2007). La idea de América Latina. La herida colonial y la opción decolonial. Barcelona: Gedisa.

Modonesi, M.; Rebón, J. (2011). Una década en movimiento. Luchas populares en América Latina (2000-2009). Buenos Aires: CLACSO.

Pageau, Christian (2017) .Pensamiento-Praxis Nacional y Popular en la producción de Fernando "Pino" Solanas (1968-2016). Tesis doctoral, Letras, Fac. Hum., UNNE.

Quijano, A. (1992). Colonialidad y modernidad/racionalidad. Perú indígena, 13(29), 11 20.

Román, M (2009). La potencialidad política de la cultura indígena americana. En: J. Lencinas, M. Román y G. Vega (comps.). Pensar lo propio. Perspectivas filosóficas en torno de lo político y social (pp. 185-201). Resistencia: El Apagón.

Sader, E. (2008). El nuevo topo. Los caminos de la izquierda latinoamericana. Buenos Aires: Siglo XXI y CLACSO. 
Torres Roggero, Jorge. (2010). Dos profetas de la des-colonialidad en Argentina. En

Zulma Palermo (comp.). Pensamiento argentino y opción decolonial. 49-85.

Buenos Aires: Ed. Del Signo, Colección El Desprendimiento.

Zibecchi, R. (2006). Dispersar el poder. Los movimientos sociales como poderes antiestatales. Buenos Aires: Tinta Limón. 